\title{
Microbial hazards in animal products
}

\author{
BY ROBERT H. MADDEN \\ Food Microbiology Research Division, Department of Agriculture for Northern Ireland, \\ Newforge Lane, Belfast BT9 5PX
}

To fully discuss microbiological hazards in animal products would be a major undertaking, hence the scope of the present overview will, of necessity, be limited. The scope of the microbiology will be reduced by concentrating on bacteria whilst the area of 'animal products' will be confined mainly to meats. Thus, dairy products will largely be excluded and meat, defined as 'flesh of animals used as food' will be the main topic of discussion. This definition excludes edible offals, further narrowing the topic under discussion, although a very large area still remains.

Locally a 'demarcation line' is drawn at the point of death of an animal in an abattoir. At this point muscle becomes meat and for research purposes the animal moves from the province of the veterinarian to that of the food scientist. However, both these research areas overlap considerably, as will be seen later. Statutory controls are another matter and these will not be considered in detail due to their complexity and variation from country to country.

By concentrating on the muscle tissue an animal can be reduced to a simple model for the purposes of this discussion. A cylinder with a tube running through it will serve as a basic shape. The tube is the gastrointestinal (GI) tract with the mouth at one end and anus at the other, whilst the cylinder surface is the animal's outer covering or external surface. The remainder of the cylinder represents the muscle required as food, or as the basis for processed products. Each of these parts will have an associated microflora which can contribute to microbial hazards. Table 1 lists some of the pathogenic genera and species associated with meat products. Note that for vulnerable individuals such as the elderly, or young children the consequences of gastroenteritis can be severe with death possible from, for example, salmonellosis (Doyle \& Cliver, 1990).

The consequences of the hazard may be a disease, which will be termed a zoonosis since the infection was transferred from an animal to man. Alternatively microorganisms may produce a toxin which is ingested and causes subsequent illness, referred to as intoxication. These two forms of disease, infection and intoxication, are simply distinguished since intoxication is caused by a toxin only and the presence of a live organism is not required once it has produced the necessary level of toxin. Infection on the other hand is caused by a live organism which must be present. Should these two forms of illness be restricted to the GI tract then they would simply be referred to as food-poisoning. Since the GI tract is tubular in nature the body's reaction to food poisoning tends to be to expel the foodstuff upwards from the stomach, i.e. vomiting occurs, or excess fluid is produced in the lumen of the lower part of the gut and diarrhoea occurs. A variety of other symptoms may also be present such as fever, abdominal pain, and malaise.

Given the limited range of symptoms and often transient nature of the illness the vast majority of food-poisoning cases go unreported and even when medical help is sought a high proportion of cases is recorded as being due to unknown causes (Cliver, 1990). This 
Table 1. Pathogens isolated from meat products

\begin{tabular}{llll}
\hline \hline Microbial group & Genus/species & Habitat & Effect on man \\
\hline Enterobacteriaceae & Arizona & GI & Gastroenteritis \\
& Edwardsiella & GI & Gastroenteritis \\
& Klebsiella & GI & Gastroenteritis \\
& Proteus & GI & Gastroenteritis \\
& Providencia & GI & Gastroenteritis \\
& Salmonella & GI & Gastroenteritis \\
& Shigella & GI & Gastroenteritis \\
Vibrios/spirillum & Aeromonas & GI & Gastroenteritis \\
& Campylobacter & E/GI & Gastroenteritis \\
Gram positive rods/cocci & GI & Gastroenteritis \\
& Vibrio & E/GI & Gastroenteritis \\
& Enteria & E/GI & Infection/death \\
Spore-forming rods & Staphylococci & GI & Gastroenteritis \\
& Bacillus cereus & S & Gastroenteritis \\
& Bacillus subtilis & E & Gastroenteritis \\
& Clostridium botulinum & E & Gastroenteritis \\
& Clostridium perfringens & GI & Intoxication/death \\
\hline \hline
\end{tabular}

E, environmental; S, external surface; GI, gastrointestinal tract.

reflects the wide variety of causative agents and the difficulties involved in isolating and characterizing them. It should also be noted that foodstuffs generally reflect the cultures of the people utilizing them, hence some hazards can be limited to specific ethnic groups or nationalities. For example intoxication due to the ingestion of puffer fish toxin is largely a Japanese phenomenon (Taylor \& Schantz, 1990) whilst an outbreak of botulism in New York was due to Russian emigrants preparing (badly) a fermented meat dish characteristic of their home. Again to limit the scope of the present article it will largely reflect the experience of hazards seen in animal products in the UK and Europe.

Returning to the 'model animal', the three main areas available for microbial colonization during the life of the animal are the muscles, the GI tract and the exterior surface. These will be considered during the growth of the animal on the farm, then on its journey to, and through, the abattoir to the final meat product. First, however, some of the more common microbiological causes of hazards in meat products will be considered in relation to their normal habitats as noted for the model animal.

\section{MICROBIAL HAZARDS ASSOCIATED WITH MEAT}

Meat

As a muscle, meat should be protected by the animal's immune system and is, therefore, normally sterile. In fact carefully excised portions of meat are used to provide a sterile medium for the growth of pure cultures of spoilage bacteria when investigating off-odours. Hence, the presence of any microbes in the muscles is indicative of disease. Under conditions of good husbandry any disease symptoms seen in an animal would result in veterinary inspection and prophylactic measures being taken. Further statutory veterinary inspections will normally occur later, hence muscle should be relatively free of 
bacterial hazards. Other hazards may be present, as some human parasites such as Taenia saginata (beef tapeworm) and Taenia solium (pork tapeworm) have life cycles involving the infection of animal muscles. However, these are rare in the UK.

For muscles to become infected an invasive pathogen is required and one of the most famous examples noted recently was Salmonella enteritidis phage type 4 . This organism is highly invasive with regard to poultry and orally applied cells could be recovered throughout the carcass of young broilers killed $24 \mathrm{~h}$ after the initial application (M. H. Hinton, personal communication). This contrasts with the majority of serotypes which would remain confined to the GI tract, although other invasive serotypes are known. For man the most serious invasive types are those causing enteric fever, Salmonella typhi and Salmonella paratyphi, better known as typhoid.

Other pathogens of note are Listeria monocytogenes, enteroinvasive Escherichia coli, and Pasteurella haemolytica. All these are characterized by the fact that they can be part of the normal flora of the animal and cause no disease, i.e. they are commensals. However, under certain conditions they can become invasive and pathogenic. Thus, it is very important to maintain checks on animal health, as postmortem detection of a pathogen may indicate infection or poor abattoir hygiene, allowing meat contamination by GI tract contents.

\section{GI tract and its associated microbial hazards}

The GI tract will vary in nature depending on the animal concerned due to evolutionary specialization, but it has the most heavily exploited microbiological niches present in, or on, an animal. The microflora of the colon and caecum can reach $10^{10}-10^{11}$ colonyforming units (cfu)/g, thus, the potential for meat contamination from this material is very high. The exact nature of the flora will depend on the specific site and animal and the age of the animal. Young chicks, for example, are particularly susceptible to infection by Campylobacter spp. (Stern \& Meinersmann, 1989) and the disease will rapidly spread through a flock of young broilers by the oral-faecal route. However, the symptoms can be mild and the chicks rapidly recover, but in many birds Campylobacter will become part of the commensal flora of the lower GI tract. Similarly in calves $E$. coli strains can cause diarrhoeal illness but then become part of the normal gut flora, with the potential to cause hazards later.

\section{Exterior surface}

The microflora of an animal's hide or feathers will reflect many factors: type of enclosure, feed composition, season of the year, and grooming habits. However, it can be expected to contain representatives of the microflora of every niche to which the animal is exposed. Thus, microbes from soil, water, the GI tract and enclosures, and air-borne microflora can be present as well as the normal surface commensals for that species. There is enormous scope for microbial contamination and this flora must, as far as possible, be kept from the meat postmortem. Again normal commensals can have hazard potential with Staphylococcus aureus, which is routinely present on skin and hair (Jay, 1992) but can produce toxins in food, being the best-known example.

There are also species which are benign in their normal habitat but which can become pathogens when their habitat is changed from, for example, rotting vegetation to 
animals. Listeria monocytogenes is one of the best examples of this kind of behaviour. Even more problematical, L. monocytogenes can adapt from its external niche to become a GI commensal; hence healthy animals can carry large numbers of this potentially lethal pathogen in their GI tract. The genus Aeromonas also contains species which are ubiquitous in the environment but are potential pathogens for a wide range of animals including man.

\section{ON THE FARM}

Animal husbandry will vary greatly depending on the animal species considered and, for a given animal, regimens can be very different. Pigs and poultry can be reared under intensive conditions and, hence, be permanently indoors, or, for example, have access to open fields. However, both are slaughtered relatively young, with broilers being aged about 6 weeks and pigs 9 months. The manner in which animals are housed will directly affect the microflora of their exterior, whilst the age at slaughter will have a major effect on the microflora of the GI tract. In the real world the three 'model habitats' will share microflora in line with the microbial ecology dictum that 'everything is everywhere, the environment selects'. Thus, examples of particular species may be widely spread by cross-contamination but they will only be metabolically active in a limited range of niches. Since meat has already been discussed (pp. 310-311), only the remaining two 'model habitats' are discussed here.

\section{Exterior surface}

The links between housing and exterior microflora are relatively obvious and are best illustrated by considering beef cattle. Animals slaughtered in summer will have spent several months in open fields, whilst those killed in winter will have spent months indoors in pens. A cursory examination of the hides would allow these two times of slaughter to be differentiated, since winter hides would be much dirtier, with considerable quantities of dung matted onto the underside of the animal. Locally we have seen microbial counts of $10^{3}-10^{5} \mathrm{cfu} / \mathrm{g}$ (after $3 \mathrm{~d}$ incubation at $22^{\circ}$ ) of hair from hides sampled in summer but $10^{8}-10^{10} \mathrm{cfu} / \mathrm{g}$ in winter. Since cattle in an abattoir will always have the hide opened first in the brisket area, i.e. just behind the front legs, there are obvious consequences for the hygienic quality of the carcass and, hence, its potential to be a subsequent hazard to man.

\section{GI tract}

The majority of the winter-hide microflora are normally previous residents of the GI tract and this microflora will reflect the age of the animal and the diet. Newborn animals have no internal microflora and are vulnerable to infection as a result. Adults possess a gut flora of commensal organisms which compete with pathogens for colonizable niches, hence adults often require much higher doses of pathogens for infection to occur. The principle of 'competitive exclusion' is now well known (Mead \& Impey, 1986) and efforts are now directed at the best method of delivering adult caecal flora to newly hatched broiler chicks, for example.

The principal benefit of competitive exclusion is that the infectious dose for a given pathogen is markedly increased, hence control is much easier. The possibility of a small 
number of cells of a pathogen arriving in a herd or flock is always present. If a single animal is infected with a GI pathogen then it will normally shed very high numbers of the organism in its faeces, exposing the rest of the animals to many times the infectious dose. This kind of event is frequently seen in broiler houses with Campylobacter with the result that virtually every bird is infected within days of the first symptoms appearing. Parallel results can be seen in any situation where young animals are intensively reared, e.g. pigs and calves.

The real world does not allow a neat segregation of the microflora, as some species, such as Listeria, can exist equally well on vegetation at $0-30^{\circ}$ or in the GI tract of sheep and cattle at $37^{\circ}$. Given the activities of wildlife on and around farms, microbes can be transported from one building to another (by insects or rodents) or even from one county to another (by birds). Thus, a Listeria sp. on the coat of a cow could be from the fields it browsed in, from faecal contamination of the herd, or could even have fallen from a passing gull.

Ultimately the animals reared for meat will normally have muscles which are microbe-free but will have well-colonized exteriors and very high numbers of organisms in the GI tract. Those animals which are intensively reared and slaughtered young will have the greatest potential for carrying pathogens.

\section{TRANSPORT TO THE ABATTOIR}

In Northern Ireland journeys from the farm to the abattoir can be a few miles but in other countries farm animals may face long journeys to the abattoir. The travel can be stressful, especially if water and food are not supplied. Further stress can be applied when animals with a social hierarchy, e.g. cattle, are mixed. These animals will then attempt to reassert their social position and the consequent fighting causes stress which can increase susceptibility to GI infections. Mixing animals in confined areas will also ensure that many opportunities for cross-contamination exist. Whilst the time period required for full-scale infection will not be available, the animals will be able to contaminate their exterior surfaces. This is especially so where tiered transport systems are used, e.g. the 'double decker' carriers for cattle and 'triple-decker' carriers for sheep. Unless the vehicles are well maintained, animals on the lower decks will be exposed to the waste products of those above, an obvious opportunity for the spread of pathogens.

More understanding of animal behaviour has led to better handling regimens on farms and in abattoirs, whilst financial pressures have seen abattoirs moved from their previous sites in centres of population to farming areas. It is usually more cost-effective to kill and process near the sites of production, then ship the high-value products on to the population centres. In the US over the past 30 years, cattle shipments to city feed lots have virtually ceased and, instead, processing is conducted near the farms with shipments of vacuum-packed beef replacing those of live cattle. Since only the carcass parts required are shipped, transport costs are reduced significantly. The processing of residual waste materials must be performed with care, however, as cycling of pathogens can occur. Processing to give feed supplements such as meat-and-bone meal must be rigorous enough to remove all pathogens, and considerable care is required to prevent the final product being re-contaminated. Recently, rapid methods for the detection of Salmonella have been developed to allow screening for this pathogen in processed animal protein (Donaghy \& Madden, 1993), hence checking on final product can be readily performed. 
The producer must totally segregate raw materials from final product in a manner analogous to a food producer serving the human population. Failures can lead to outbreaks of salmonellosis, or worse still bovine spongiform encephalopathy.

\section{PRIMARY PROCESSING}

Most animals are held first in the lairage, and are then taken to slaughter. Again this can be an excellent cross-contamination opportunity. Once the slaughter procedures are running cross-contamination is inevitable. The worst examples will occur with poultry. Returning to the basic model animal it can be seen that poststunning the aim is to remove the outer covering, then the GI tract, whilst attempting to retain the 'clean' state of the muscles. However, poultry are rather small, hence automated processing is conducted whenever possible. The large number of mechanized operations removes the opportunity for a human operator's delicacy of touch, and the high line speeds, 1000 birds per $h$ is not uncommon, compound the problems.

After stunning using high-voltage electricity birds have their throats cut and die by exsanguination. Since one cutting tool cuts all the birds any pathogen present will contaminate several of those following. The feathers must then be removed, so they are softened by dragging the birds through a scald bath held at about $50^{\circ}$. This is not sufficiently hot to kill pathogens, hence it will again permit cross-contamination (Thomas et al. 1986). Once out of the bath plucking is performed by rapidly rotating rubber fingers which pull the feathers from the follicles, which can then be inoculated by the remaining scald-bath liquid. The rubber of the pluckers can become cracked and microbes can then colonize the spaces. Strains of Staphylococcus atireus have been shown to adapt to this habitat but, fortunately, those adapting were not pathogenic (Thompson \& Patterson, 1983). Virtually all birds passing through the pluckers emerged with $S$. aureus contamination, illustrating the potential problems for cross-contamination that mechanization can bring.

However, it is later, during evisceration, that most problems arise. As the number of GI tract pathogens is high, careful evisceration is essential to ensure the carcass does not become contaminated. With poultry this is not possible and with mechanical evisceration some breakage is to be expected. Thus, should Campylobacter be present as a commensal, large numbers will be deposited on the carcass and the equipment, leading to following birds also being contaminated. Hence, poultry are associated most with GI pathogens such as Salmonella and Campylobacter.

Larger animals such as cattle and pigs can be handled by human operators and, if due care is taken, opportunities for GI contamination of the carcass should be minimized. Also veterinary inspections of the animals in the lairage, followed by inspections during the evisceration procedure, should lead to the condemnation of soiled carcasses. The parasites mentioned previously should be detected, and appropriate measures taken to condemn contaminated carcasses.

It should also be noted that the workers can contaminate the carcasses and vice-versa. Lack of personal hygiene, or failure to properly clean equipment can lead to pathogens being applied to the meat surface. This can be compounded by setting the evisceration line speed too high, in an effort to maximize throughput. Hence, carcasses can be exposed to hazards from a wide range of sources, but if they are cooled properly virtually no growth of pathogens will occur due to the temperatures involved, $4-10^{\circ}$, and due to 
the competing microflora present on the meat surface. Exposed surfaces will also tend to dry which will control the growth of bacteria, most of which require a relatively high water activity. After cooling processing proper can begin.

\section{SECONDARY PROCESSING}

The extensive range of processes available means that they cannot be covered in any detail, but a brief glimpse of possible problems can be made by grouping processes according to the stress they apply to micro-organisms.

\section{Little or no stress}

Chilling and cool smoking would fall into this category, and products such as raw pork mince (eaten in Germany) or steak tartare would be examples of final products with no further processing. The consumer would be exposed to all the hazards noted previously. Undercooked hamburgers will also expose consumers to these hazards and an outbreak of haemolytic $E$. coli in England was traced to lightly cooked beefburgers. Parasites can be spread by such products unless freezing has occurred. For most bacteria freezing to $-20^{\circ}$ is a modest stress but parasitic resting stages can be killed by appropriate exposure; Trichinella held at $-15^{\circ}$ for about $20 \mathrm{~d}$ will lose viability.

Campylobacter are unusual in that they are killed by freezing and the upsurge in cases of this organism has been linked by some to the market for poultry products moving from frozen products to chilled products.

\section{Moderate stress}

Cooking and curing will kill many pathogens, given adequate treatments. However, this then means the product is open to subsequent colonization due to either crosscontamination or from the food handler's microflora. Pies can be re-infected with Salmonella via, for example, aspic glaze and food held below $55^{\circ}$ is at risk from spores of Clostridium perfringens which can then germinate and rapidly increase to infective levels. Carvery roasts and meat products held in poorly maintained ovens are particularly susceptible to the latter pathogen.

\section{Severe stress}

Canning of meat is carefully regulated due to the danger of the lethal pathogen Clostridium botulinum which is widespread in soil. This is also a sporing organism, hence canning is designed either to destroy the spores or to work in conjunction with other prophylactic measures such as the addition of nitrite which prevents successful outgrowth from spores. Problems only arise if processing is faulty or the product is exposed to pathogens after opening the container. An example of the consequences of faulty canning occurred in Aberdeen, Scotland in the early 1960s when Argentinean corned beef from a wholesale can was sold. A typhoid epidemic resulted and was traced to a leak in the can. Cooling water in the processing plant should have been chlorinated but was not, allowing Salmonella typhi access from the local water supply to the final product. During transport growth to the infectious dose level occurred. 


\section{CONCLUSIONS}

Meat products are prepared from the carcasses of animals which may harbour parasites whose specific host is man, or with which man shares sufficient similarity to allow successful infection. With microbes a wide host range is often seen, allowing organisms like Campylobacter, Salmonella and Listeria to cause pathogenesis in livestock and, subsequently, man. Good husbandry on the farm and care in primary processing will minimize the potential for subsequent human infection, but the potential for hazards will always be present. Once processing enters the equation the opportunities for widespread dissemination of hazardous meat products rise markedly. Thus, the production, transport and sale of meat products must be performed with the utmost care and preferably be subject to a hazard analysis critical control point evaluation. Following the correct implementation of such a scheme meat products should not present any undue hazard.

\section{REFERENCES}

Cliver, D. O. (editor) (1990). Transmission of diseases via foods. In Foodborne Diseases, pp. 3-15. San Diego: Academic Press Inc.

Donaghy, J. \& Madden, R. H. (1993). Detection of Salmonella in animal protein by Rappaport-Vassiliadis broth using indirect impediometry. International Journal of Food Microbiology 17, 281-288.

Doyle, M. P. \& Cliver, D. O. (1990). Salmonella. In Foodborne Diseases, pp. 185-204 [D. O. Cliver, editor]. San Diego: Academic Press Inc.

Jay, J. M. (1992). Modern Food Microbiology, 4th ed. London: Chapman and Hall.

Mead, G. C. \& Impey, C. S. (1986). The present status of the Nurmi Concept for reducing carriage of food-poisoning salmonellae and other pathogens in live poultry. In Elimination of Pathogenic Organisms from Meat and Poultry, pp. 57-77 [F. J. M. Smulders, editor]. Amsterdam: Elsevier.

Stern, N. J. \& Meinersmann, R. J. (1989). Potentials for colonization control of Campylobacter jejuni in the chicken. Journal of Food Protection 55, 427-430.

Taylor, S. L. \& Schantz, E. J. (1990). Naturally occurring toxicants in foods. In Foodborne Diseases, pp. 67-84 [D. O. Cliver. editor]. San Diego: Academic Press Inc.

Thomas, C. J., McMeekin, T. A. \& Patterson, J. T. (1986). Prevention of microbial contamination in the poultry processing plant. In Elimination of Pathogenic Organisms from Meat and Poultry, pp. 163-179 [F. J. M. Smulders, editor]. Amsterdam: Elsevier.

Thompson, J. K. \& Patterson, J. T. (1983). Staphylococcus aureus from a site of contamination in a broiler processing plant. Record of Agricultural Research 31, 45-53. 